Supporting information for

\title{
Magnetism-Vanishing Stabilizes the Pyrite-Type 3d Transition Metal Peroxides at High Pressures
}

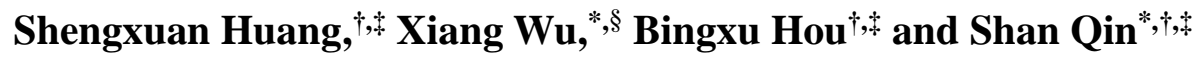

†Key Laboratory of Orogenic Belts and Crustal Evolution, MOE, Peking University, Beijing 100871, China

\$School of Earth and Space Sciences, Peking University, Beijing 100871, China

${ }^{\S}$ State Key Laboratory of Geological Processes and Mineral Resources, China University

of Geosciences (Wuhan), Wuhan 430074, China

\section{Corresponding Authors}

"E-mail: Xiang Wu (wuxiang@cug.edu.cn)

*E-mail: Shan Qin (sqin@ pku.edu.cn) 
Figure S1

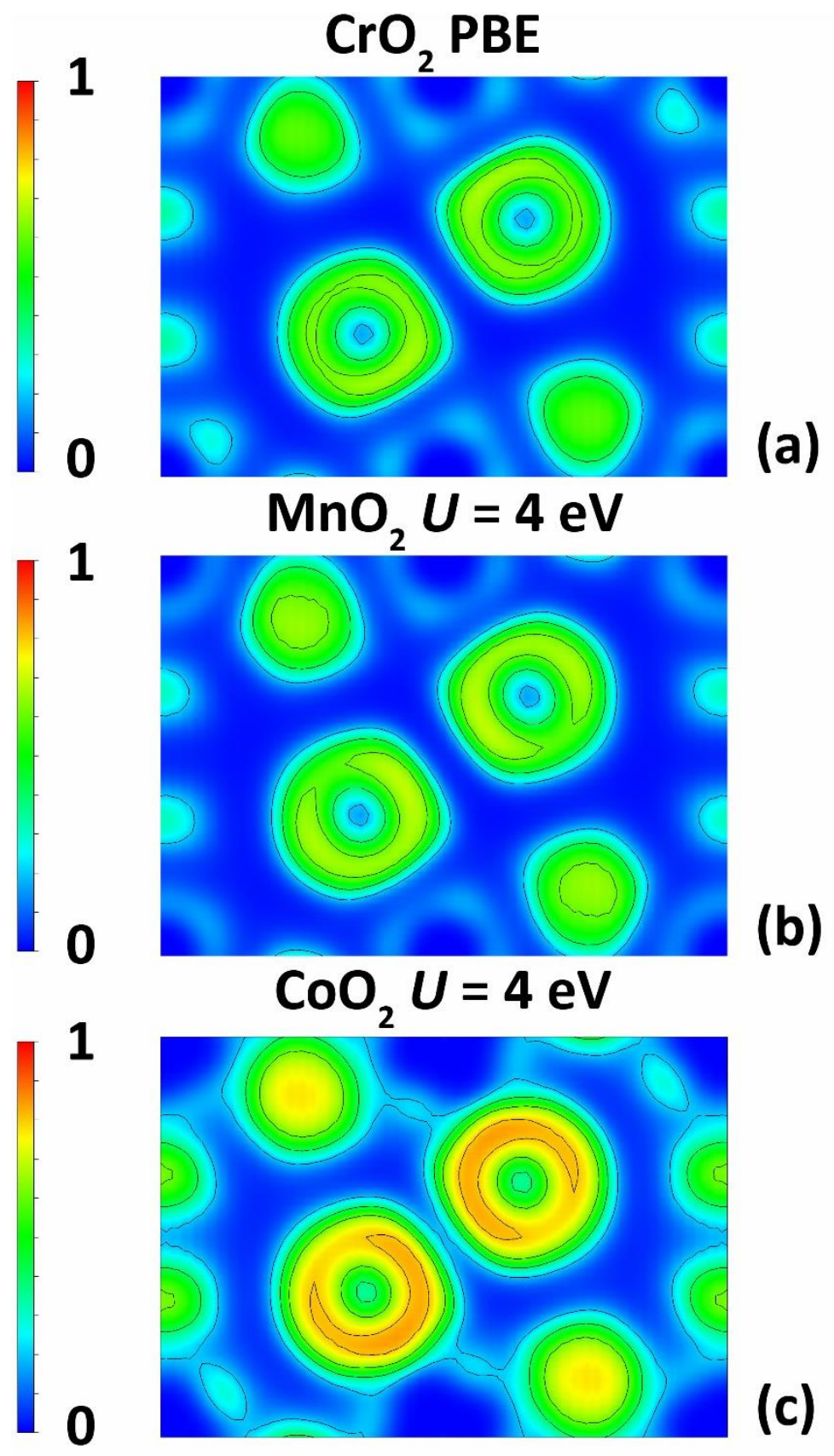

Figure S1 The electron localization function (ELF) of pyrite-type $\mathrm{TMO}_{2}$ at $150 \mathrm{GPa}$ by PBE or PBE + $\boldsymbol{U}$ methods. The section cut is through the (1 $\overline{1} 0)$ plane. 


\section{Figure S2}

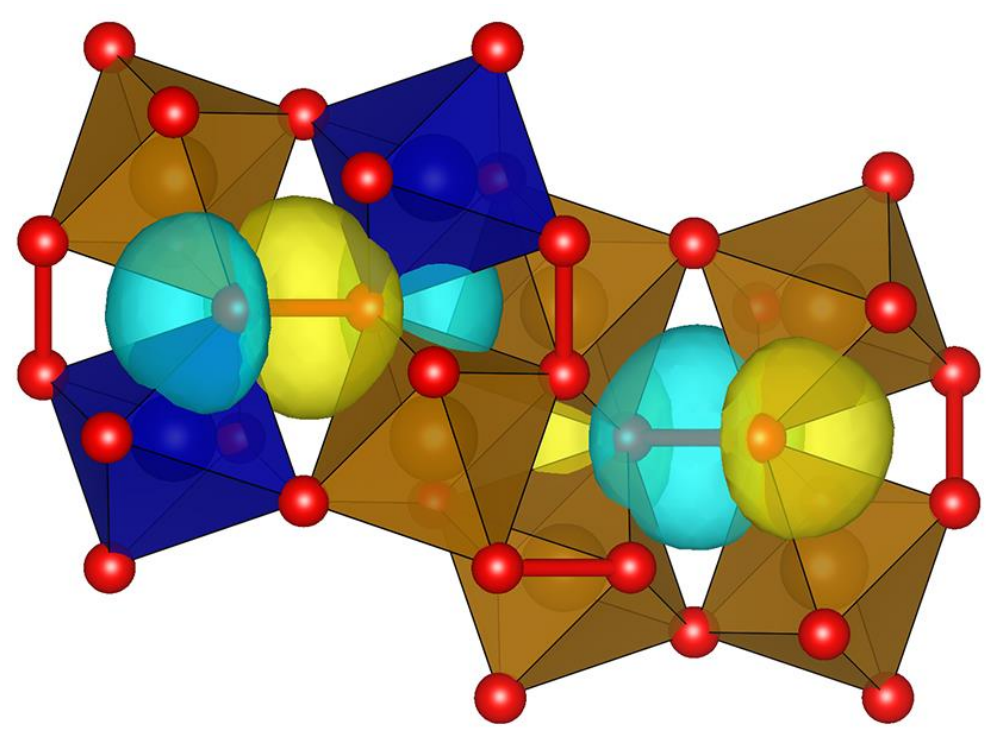

Figure $\mathrm{S} 2$ The O-O chemical bonding in pyrite-type Co-doped $\mathrm{FeO}_{2}$ by the Solid State Adaptive Natural Density Partitioning (SSAdNDP) method. The brown, blue and red spheres represent $\mathrm{Fe}, \mathrm{Co}$ and $\mathrm{O}$, respectively. 
Figure S3
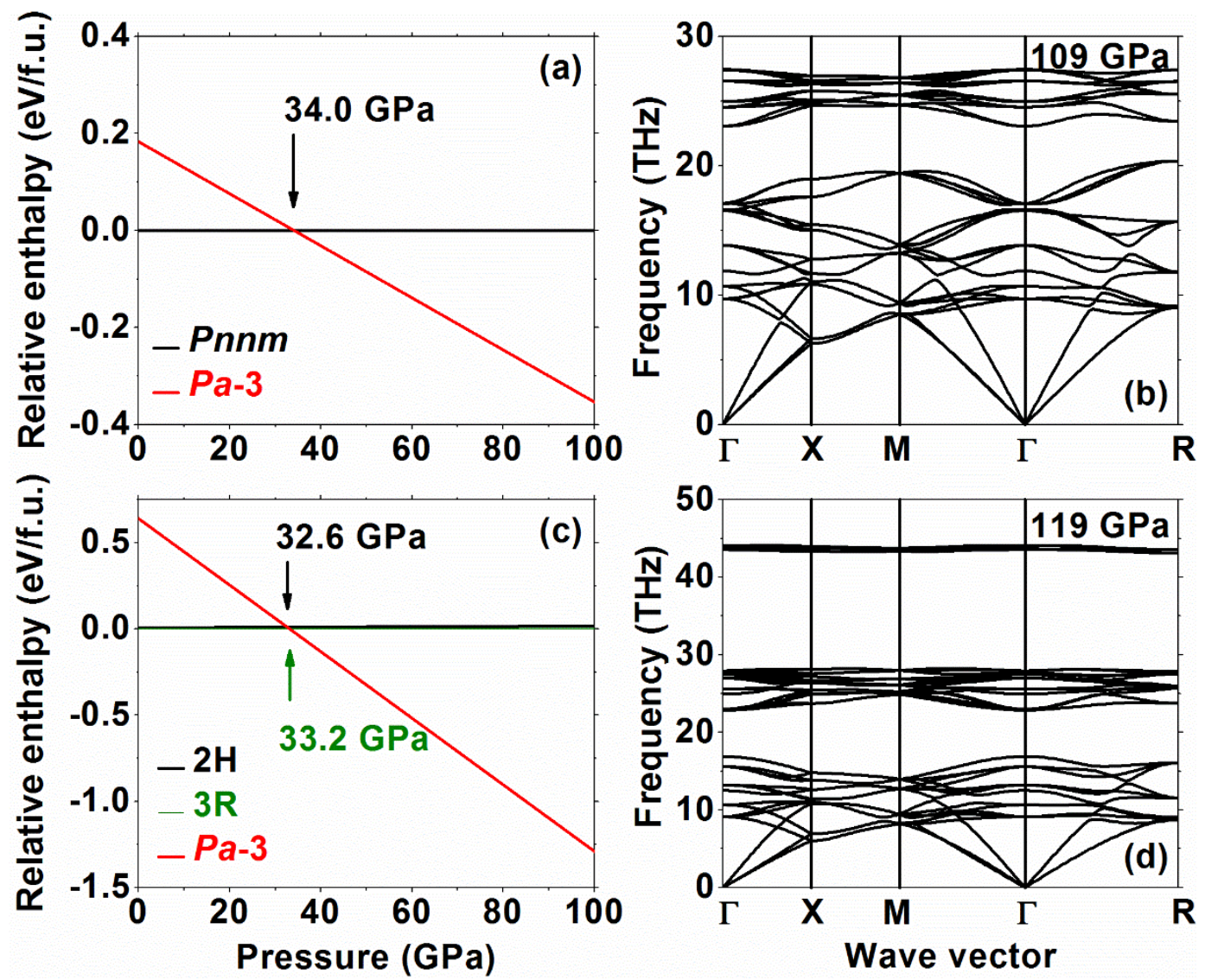

Figure S3 Pressure-induced structural transitions and dynamical stability of $\mathrm{CoO}_{2}$ and $\mathrm{CoO}_{2} \mathrm{H}$ at high pressures. (a) Relative enthalpy of $\mathrm{CaCl}_{2}$-type (Pnnm) and pyritetype $\mathrm{CoO}_{2}$ as a function of pressure. (b) The phonon dispersion of pyrite-type $\mathrm{CoO}_{2}$ at 109 GPa. (c) Relative enthalpy of 2H-type, 3R-type and pyrite-type $\mathrm{CoO}_{2} \mathrm{H}$ as a function of pressure. (d) The phonon dispersion of pyrite-type $\mathrm{CoO}_{2} \mathrm{H}$ at $119 \mathrm{GPa}$. 
Table S1 Volume-energy data of pyrite-type $\mathrm{CrO}_{2}, \mathrm{MnO}_{2}$ and $\mathrm{CoO}_{2}$ with various initial magnetic arrangements by $\mathrm{PBE}$ or PBE $+U$ methods.

\begin{tabular}{|c|c|c|c|c|c|c|c|}
\hline$V\left(\AA^{3}\right)$ & $E(\mathrm{eV})$ & $V\left(\AA^{3}\right)$ & $E(\mathrm{eV})$ & $V\left(\AA^{3}\right)$ & $E(\mathrm{eV})$ & $V\left(\AA^{3}\right)$ & $E(\mathrm{eV})$ \\
\hline \multicolumn{8}{|c|}{$\mathrm{CrO}_{2}(\mathrm{PBE})$} \\
\hline \multicolumn{2}{|c|}{$\mathrm{FM}_{-}+4$} & \multicolumn{2}{|c|}{ AFM_+4 } & \multicolumn{2}{|c|}{ FM_+2 } & \multicolumn{2}{|c|}{$\mathrm{NM}$} \\
\hline 115.57 & -98.485 & 115.57 & -97.719 & 122.61 & -92.732 & 108.78 & -95.941 \\
\hline 112.14 & -98.754 & 112.14 & -97.974 & 119.00 & -92.894 & 105.48 & -96.073 \\
\hline 108.78 & -98.890 & 108.78 & -98.101 & 115.47 & -92.932 & 102.25 & -96.041 \\
\hline 105.48 & -98.877 & 105.48 & -98.081 & 112.00 & -92.833 & 99.09 & -95.825 \\
\hline 102.25 & -98.698 & 102.25 & -97.900 & 108.61 & -92.581 & 95.99 & -95.406 \\
\hline 99.09 & -98.332 & 99.09 & -97.537 & 105.28 & -92.159 & 92.96 & -94.761 \\
\hline 95.99 & -97.759 & 95.99 & -96.974 & 102.03 & -91.551 & 89.99 & -93.866 \\
\hline 92.96 & -96.955 & 92.96 & -96.187 & 98.84 & -90.736 & 87.09 & -92.695 \\
\hline 89.99 & -95.895 & 89.99 & -95.154 & 95.72 & -89.692 & 84.25 & -91.218 \\
\hline 87.09 & -94.552 & 87.09 & -93.849 & 92.66 & -88.394 & 81.48 & -89.404 \\
\hline 84.25 & -92.897 & 84.25 & -92.243 & 89.68 & -86.817 & 78.76 & -87.217 \\
\hline 81.48 & -90.899 & 81.48 & -90.304 & 86.75 & -84.933 & & \\
\hline \multirow[t]{2}{*}{78.76} & -88.526 & 78.76 & -87.998 & 83.89 & -82.710 & & \\
\hline & & & & 81.10 & -80.114 & & \\
\hline \multicolumn{8}{|c|}{$\mathrm{CrO}_{2}(U=3 \mathrm{eV})$} \\
\hline \multicolumn{2}{|c|}{$\mathrm{FM}+4$} & \multicolumn{2}{|c|}{ AFM_+4 } & \multicolumn{2}{|c|}{ FM_+2 } & \multicolumn{2}{|c|}{$\mathrm{NM}$} \\
\hline 115.57 & -91.727 & 119.07 & -90.638 & 126.28 & -87.846 & 112.14 & -85.321 \\
\hline 112.14 & -91.865 & 115.57 & -90.871 & 122.61 & -88.060 & 108.78 & -85.555 \\
\hline 108.78 & -91.873 & 112.14 & -90.989 & 119.00 & -88.160 & 105.48 & -85.640 \\
\hline 105.48 & -91.735 & 108.78 & -90.978 & 115.47 & -88.133 & 102.25 & -85.559 \\
\hline 102.25 & -91.434 & 105.48 & -90.821 & 112.00 & -87.966 & 99.09 & -85.295 \\
\hline 99.09 & -90.949 & 102.25 & -90.501 & 108.61 & -87.643 & 95.99 & -84.826 \\
\hline 95.99 & -90.261 & 99.09 & -89.998 & 105.28 & -87.147 & 92.96 & -84.130 \\
\hline 92.96 & -89.345 & 95.99 & -89.292 & 102.03 & -86.462 & 89.99 & -83.184 \\
\hline 89.99 & -88.176 & 92.96 & -88.359 & 98.84 & -85.567 & 87.09 & -81.962 \\
\hline 87.09 & -86.727 & 89.99 & -87.175 & 95.72 & -84.440 & 84.25 & -80.437 \\
\hline 84.25 & -84.969 & 87.09 & -85.713 & 92.66 & -83.058 & 81.48 & -78.576 \\
\hline 81.48 & -82.869 & 84.25 & -83.944 & 89.68 & -81.395 & 78.76 & -76.347 \\
\hline \multirow[t]{3}{*}{78.76} & -80.392 & 81.48 & -81.837 & 86.75 & -79.423 & & \\
\hline & & 78.76 & -79.358 & 83.89 & -77.113 & & \\
\hline & & & & 81.10 & -74.431 & & \\
\hline
\end{tabular}




\section{Continued Table S1}

\begin{tabular}{|c|c|c|c|c|c|c|c|}
\hline$V\left(\AA^{3}\right)$ & $E(\mathrm{eV})$ & $V\left(\AA^{3}\right)$ & $E(\mathrm{eV})$ & $V\left(\AA^{3}\right)$ & $E(\mathrm{eV})$ & $V\left(\AA^{3}\right)$ & $E(\mathrm{eV})$ \\
\hline \multicolumn{8}{|c|}{$\mathrm{MnO}_{2}(U=6 \mathrm{eV})$} \\
\hline \multicolumn{2}{|c|}{ FM_+4 } & \multicolumn{2}{|c|}{ AFM_+4 } & \multicolumn{2}{|c|}{ FM_+2_HS } & \multicolumn{2}{|c|}{ AFM_+2_HS } \\
\hline 126.29 & -80.731 & 122.36 & -79.948 & 138.00 & -77.994 & 130.11 & -79.621 \\
\hline 122.65 & -80.899 & 118.86 & -80.178 & 134.02 & -78.234 & 126.29 & -79.787 \\
\hline 119.07 & -80.996 & 115.43 & -80.334 & 130.11 & -78.392 & 122.54 & -79.864 \\
\hline 115.57 & -81.013 & 112.07 & -80.403 & 126.29 & -78.456 & 118.86 & -79.843 \\
\hline 112.14 & -80.942 & 108.78 & -80.370 & 122.54 & -78.415 & 115.26 & -79.706 \\
\hline 108.78 & -80.769 & 105.55 & -80.218 & 118.86 & -78.255 & & \\
\hline 105.48 & -80.485 & 102.38 & -79.928 & 115.26 & -77.963 & & \\
\hline 102.25 & -80.071 & 99.28 & -79.481 & 111.73 & -77.524 & & \\
\hline 99.09 & -79.511 & 96.24 & -78.857 & 108.28 & -76.921 & & \\
\hline 95.99 & -78.780 & 93.26 & -78.035 & 104.89 & -76.136 & & \\
\hline 92.96 & -77.852 & 90.35 & -76.991 & 101.58 & -75.149 & & \\
\hline 89.99 & -76.697 & 87.49 & -75.701 & 98.34 & -73.939 & & \\
\hline 87.09 & -75.284 & 84.70 & -74.139 & 95.17 & -72.482 & & \\
\hline 84.25 & -73.581 & 81.97 & -72.277 & 92.06 & -70.753 & & \\
\hline 81.48 & -71.556 & 79.30 & -70.087 & 89.03 & -68.725 & & \\
\hline \multirow[t]{2}{*}{78.76} & -69.174 & & & 86.06 & -66.368 & & \\
\hline & & & & 83.16 & -63.649 & & \\
\hline \multicolumn{2}{|c|}{ FM_+2_LS } & \multicolumn{2}{|c|}{ AFM_+2_LS } & \multicolumn{2}{|c|}{ NM } & & \\
\hline 115.57 & -72.168 & 104.89 & -80.171 & 111.95 & -67.809 & & \\
\hline 112.14 & -72.428 & 101.58 & -79.830 & 108.68 & -68.118 & & \\
\hline 108.78 & -72.577 & & & 105.49 & -68.305 & & \\
\hline 105.48 & -72.603 & & & 102.36 & -68.352 & & \\
\hline 102.25 & -72.490 & & & 99.29 & -68.247 & & \\
\hline 99.09 & -72.223 & & & 96.28 & -67.971 & & \\
\hline 95.99 & -71.784 & & & 93.33 & -67.507 & & \\
\hline 92.96 & -71.155 & & & 90.44 & -66.834 & & \\
\hline 89.99 & -70.315 & & & 87.62 & -65.932 & & \\
\hline 87.09 & -69.239 & & & 84.85 & -64.777 & & \\
\hline 84.25 & -67.902 & & & 82.14 & -63.344 & & \\
\hline 81.48 & -66.273 & & & 79.49 & -61.605 & & \\
\hline 78.76 & -64.323 & & & 76.90 & -59.531 & & \\
\hline 76.10 & -62.015 & & & 74.37 & -57.090 & & \\
\hline
\end{tabular}




\section{Continued Table S1}

\begin{tabular}{|c|c|c|c|c|c|c|c|}
\hline$V\left(\AA^{3}\right)$ & $E(\mathrm{eV})$ & $V\left(\AA^{3}\right)$ & $E(\mathrm{eV})$ & $V\left(\AA^{3}\right)$ & $E(\mathrm{eV})$ & $V\left(\AA^{3}\right)$ & $E(\mathrm{eV})$ \\
\hline \multicolumn{8}{|c|}{$\mathrm{MnO}_{2}(U=4 \mathrm{eV})$} \\
\hline \multicolumn{2}{|c|}{ FM_+4 } & \multicolumn{2}{|c|}{ AFM_+4 } & \multicolumn{2}{|c|}{ FM_+2_HS } & \multicolumn{2}{|c|}{ AFM_+2_HS } \\
\hline 119.07 & -85.083 & 118.88 & -84.723 & 130.04 & -81.499 & 122.73 & -85.165 \\
\hline 115.57 & -85.258 & 115.45 & -85.008 & 126.25 & -81.622 & 119.68 & -85.033 \\
\hline 113.85 & -85.312 & 112.09 & -85.197 & 122.54 & -81.641 & & \\
\hline 112.14 & -85.342 & 108.79 & -85.275 & 118.90 & -81.543 & & \\
\hline 108.78 & -85.324 & 105.56 & -85.228 & 115.33 & -81.314 & & \\
\hline 105.48 & -85.189 & 102.39 & -85.037 & 111.84 & -80.939 & & \\
\hline 102.25 & -84.912 & 99.29 & -84.684 & 108.41 & -80.401 & & \\
\hline 99.09 & -84.473 & 96.25 & -84.152 & 105.06 & -79.685 & & \\
\hline 95.99 & -83.849 & 93.27 & -83.420 & 101.78 & -78.768 & & \\
\hline 92.96 & -83.016 & 90.36 & -82.466 & 98.56 & -77.631 & & \\
\hline 89.99 & -81.944 & 87.51 & -81.266 & 95.42 & -76.252 & & \\
\hline 87.09 & -80.605 & 84.71 & -79.795 & 92.34 & -74.605 & & \\
\hline 84.25 & -78.970 & 81.98 & -78.025 & 89.33 & -72.663 & & \\
\hline 81.48 & -77.008 & 79.31 & -75.929 & 86.38 & -70.397 & & \\
\hline 78.76 & -74.685 & 76.69 & -73.473 & 83.50 & -67.775 & & \\
\hline \multicolumn{2}{|c|}{ FM_+2_LS } & \multicolumn{2}{|c|}{ AFM_+2_LS } & \multicolumn{2}{|c|}{$\mathrm{NM}$} & & \\
\hline 108.68 & -79.273 & 79.42 & -75.742 & 105.46 & -76.387 & & \\
\hline 105.48 & -79.421 & 76.89 & -73.677 & 102.36 & -76.493 & & \\
\hline 102.35 & -79.439 & & & 99.32 & -76.450 & & \\
\hline 99.28 & -79.312 & & & 96.34 & -76.241 & & \\
\hline 96.27 & -79.023 & & & 93.42 & -75.849 & & \\
\hline 93.32 & -78.551 & & & 90.56 & -75.256 & & \\
\hline 90.44 & -77.875 & & & 87.76 & -74.439 & & \\
\hline 87.61 & -76.972 & & & 85.02 & -73.376 & & \\
\hline 84.84 & -75.816 & & & 82.33 & -72.042 & & \\
\hline 82.14 & -74.382 & & & 79.71 & -70.411 & & \\
\hline 79.49 & -72.641 & & & 77.13 & -68.454 & & \\
\hline 76.89 & -70.560 & & & 74.62 & -66.138 & & \\
\hline 74.36 & -68.108 & & & & & & \\
\hline
\end{tabular}




\section{Continued Table S1}

\begin{tabular}{|c|c|c|c|c|c|c|c|}
\hline$V\left(\AA^{3}\right)$ & $E(\mathrm{eV})$ & $V\left(\AA^{3}\right)$ & $E(\mathrm{eV})$ & $V\left(\AA^{3}\right)$ & $E(\mathrm{eV})$ & $V\left(\AA^{3}\right)$ & $E(\mathrm{eV})$ \\
\hline \multicolumn{8}{|c|}{$\mathrm{CoO}_{2}(U=6 \mathrm{eV})$} \\
\hline \multicolumn{2}{|c|}{ FM_HS_+4 } & \multicolumn{2}{|c|}{ FM_HS_+2 } & \multicolumn{2}{|c|}{ AFM_HS_+2 } & \multicolumn{2}{|c|}{ FM_LS_+2 } \\
\hline 123.43 & -59.849 & 120.43 & -65.673 & 119.49 & -65.820 & 109.00 & -61.737 \\
\hline 120.27 & -59.878 & 117.09 & -65.696 & 116.43 & -65.835 & 106.18 & -62.007 \\
\hline 117.16 & -59.851 & 113.81 & -65.633 & 113.42 & -65.778 & 103.41 & -62.200 \\
\hline 114.10 & -59.758 & 110.59 & -65.476 & 110.46 & -65.642 & 100.69 & -62.309 \\
\hline 111.10 & -59.592 & 107.43 & -65.215 & 107.55 & -65.419 & 98.02 & -62.322 \\
\hline 108.15 & -59.342 & 104.33 & -64.839 & 104.70 & -65.102 & 95.39 & -62.231 \\
\hline 105.25 & -58.998 & 101.29 & -64.337 & 101.89 & -64.683 & 92.81 & -62.024 \\
\hline 102.41 & -58.548 & 98.31 & -63.696 & 99.14 & -64.151 & 90.28 & -61.690 \\
\hline 99.610 & -57.980 & 95.39 & -62.902 & 96.43 & -63.497 & 87.80 & -61.216 \\
\hline 96.87 & -57.281 & 92.53 & -61.941 & 93.78 & -62.709 & 85.36 & -60.588 \\
\hline 94.18 & -56.438 & 89.72 & -60.797 & 91.18 & -61.779 & 82.96 & -59.792 \\
\hline 91.54 & -55.434 & 86.98 & -59.451 & 88.62 & -60.691 & 80.62 & -58.811 \\
\hline 88.95 & -54.254 & 84.29 & -57.886 & 86.11 & -59.435 & 78.31 & -57.629 \\
\hline 86.41 & -52.881 & 81.65 & -56.079 & 83.65 & -57.994 & 76.05 & -56.227 \\
\hline 83.92 & -51.299 & 79.08 & -54.010 & 81.24 & -56.356 & 73.84 & -54.584 \\
\hline 81.47 & -49.488 & 76.55 & -51.655 & 78.87 & -54.504 & 71.67 & -52.679 \\
\hline 79.08 & -47.429 & & & 76.55 & -52.423 & & \\
\hline \multicolumn{8}{|c|}{ AFM_LS_+2 } \\
\hline 109.00 & -61.669 & & & & & & \\
\hline 106.18 & -61.947 & & & & & & \\
\hline 103.41 & -62.148 & & & & & & \\
\hline 100.69 & -62.263 & & & & & & \\
\hline 98.02 & -62.282 & & & & & & \\
\hline 95.39 & -62.194 & & & & & & \\
\hline 92.81 & -61.992 & & & & & & \\
\hline 90.28 & -61.661 & & & & & & \\
\hline 87.80 & -61.190 & & & & & & \\
\hline 85.36 & -60.566 & & & & & & \\
\hline 82.96 & -59.772 & & & & & & \\
\hline 80.62 & -58.794 & & & & & & \\
\hline 78.31 & -57.614 & & & & & & \\
\hline 76.05 & -56.216 & & & & & & \\
\hline 73.84 & -54.580 & & & & & & \\
\hline 71.67 & -52.684 & & & & & & \\
\hline
\end{tabular}




\section{Continued Table S1}

\begin{tabular}{|c|c|c|c|c|c|c|c|}
\hline$V\left(\AA^{3}\right)$ & $E(\mathrm{eV})$ & $V\left(\AA^{3}\right)$ & $E(\mathrm{eV})$ & $V\left(\AA^{3}\right)$ & $E(\mathrm{eV})$ & $V\left(\AA^{3}\right)$ & $E(\mathrm{eV})$ \\
\hline \multicolumn{8}{|c|}{$\mathrm{CoO}_{2}(U=4 \mathrm{eV})$} \\
\hline \multicolumn{2}{|c|}{ FM_HS_+4 } & \multicolumn{2}{|c|}{ FM_HS_+2 } & \multicolumn{2}{|c|}{ AFM_HS_+2 } & \multicolumn{2}{|c|}{ FM_LS_+2 } \\
\hline 131.42 & -62.925 & 130.96 & -66.925 & 119.49 & -67.744 & 107.43 & -66.927 \\
\hline 127.63 & -63.173 & 127.22 & -67.190 & 116.43 & -67.787 & 104.33 & -67.234 \\
\hline 123.91 & -63.352 & 123.55 & -67.386 & 113.42 & -67.758 & 101.29 & -67.436 \\
\hline 120.27 & -63.450 & 119.96 & -67.503 & 110.46 & -67.652 & 98.31 & -67.520 \\
\hline 116.69 & -63.455 & 116.43 & -67.533 & 107.55 & -67.460 & 95.39 & -67.472 \\
\hline 113.19 & -63.354 & 112.97 & -67.465 & 104.70 & -67.175 & 92.53 & -67.275 \\
\hline 109.76 & -63.134 & 109.58 & -67.287 & 101.89 & -66.789 & 89.72 & -66.913 \\
\hline 106.40 & -62.778 & 106.26 & -66.989 & 99.14 & -66.291 & 86.98 & -66.366 \\
\hline 103.11 & -62.270 & 103.01 & -66.555 & 96.43 & -65.673 & 84.29 & -65.613 \\
\hline 99.89 & -61.592 & 99.82 & -65.970 & 93.78 & -64.923 & 81.65 & -64.632 \\
\hline 96.74 & -60.723 & 96.70 & -65.218 & 91.18 & -64.033 & 79.08 & -63.399 \\
\hline 93.65 & -59.641 & 93.65 & -64.280 & 88.62 & -62.990 & 76.55 & -61.885 \\
\hline 90.63 & -58.323 & 90.66 & -63.137 & 86.11 & -61.775 & 74.08 & -60.063 \\
\hline 87.67 & -56.743 & 87.74 & -61.765 & 83.65 & -60.403 & 71.67 & -57.900 \\
\hline 84.78 & -54.873 & 84.88 & -60.141 & & & & \\
\hline 81.96 & -52.682 & 82.08 & -58.238 & & & & \\
\hline 79.19 & -50.139 & & & & & & \\
\hline \multicolumn{8}{|c|}{ AFM_LS_+2 } \\
\hline 103.40 & -67.255 & & & & & & \\
\hline 100.68 & -67.411 & & & & & & \\
\hline 98.01 & -67.469 & & & & & & \\
\hline 95.39 & -67.418 & & & & & & \\
\hline 92.81 & -67.247 & & & & & & \\
\hline 90.28 & -66.944 & & & & & & \\
\hline 87.79 & -66.496 & & & & & & \\
\hline 85.35 & -65.888 & & & & & & \\
\hline 82.96 & -65.105 & & & & & & \\
\hline 80.61 & -64.130 & & & & & & \\
\hline 78.31 & -62.945 & & & & & & \\
\hline 76.05 & -61.530 & & & & & & \\
\hline 73.84 & -59.867 & & & & & & \\
\hline 71.66 & -57.929 & & & & & & \\
\hline
\end{tabular}


Table S2 Calculated parameters of the third-order Birch-Murnaghan equation of state (energy $E_{0}$, volume $V_{0}$, bulk modulus $K_{0}$ and its pressure derivative $K_{0}$ ' at zero pressure) of pyrite-type $\mathrm{CrO}_{2}, \mathrm{MnO}_{2}$ and $\mathrm{CoO}_{2}$ with various initial magnetic arrangements. FM, AFM and NM represent the ferromagnetic, antiferromagnetic and non-magnetic states, respectively. HS and LS represent the high-spin and low-spin states, respectively.

\begin{tabular}{lllllll}
\hline Phase & Configurations & $E_{0}(\mathrm{eV})$ & $V_{0}\left(\AA^{3}\right)$ & $K_{0}(\mathrm{GPa})$ & $K_{0}$ & Methods \\
\hline $\mathrm{CrO}_{2}$ & FM_+4 & -98.907 & 107.38 & 249.3 & 4.42 & PBE \\
& AFM_+4 & -98.113 & 107.55 & 239.7 & 4.42 & \\
& FM_+2 & -92.936 & 116.21 & 203.3 & 4.39 & \\
& NM & -96.082 & 104.44 & 272.7 & 4.33 & \\
& FM_+4 & -91.889 & 110.20 & 220.8 & 4.55 & $U=3 \mathrm{eV}$ \\
& AFM_+4 & -91.005 & 110.75 & 214.5 & 4.57 & \\
& FM_+2 & -88.167 & 117.92 & 197.6 & 4.32 & \\
& NM & -85.642 & 105.38 & 265.6 & 4.29 & \\
\hline $\mathrm{MnO}_{2}$ & FM_+4 & -81.011 & 116.49 & 127.8 & 5.85 & $U=6 \mathrm{eV}$ \\
& AFM_+4 & -80.421 & 111.51 & 180.3 & 4.96 & \\
& FM_+2_HS & -78.463 & 125.83 & 153.8 & 4.50 & \\
& AFM_+2_HS & -79.869 & 121.44 & 148.6 & 5.34 & \\
& FM_+2_LS & -72.610 & 106.47 & 212.0 & 4.49 & \\
& NM & -68.356 & 102.91 & 257.5 & 4.30 & \\
& FM_+4 & -85.358 & 110.92 & 178.5 & 5.32 & $U=4 \mathrm{eV}$ \\
& AFM_+4 & -85.284 & 108.40 & 214.9 & 4.56 & \\
& FM_+2_HS & -81.652 & 123.74 & 166.6 & 4.45 & \\
& FM_+2_LS & -79.452 & 103.44 & 242.0 & 4.54 & \\
& NM & -76.497 & 101.65 & 268.5 & 4.28 & \\
\hline $\mathrm{CoO}_{2}$ & FM_HS_+4 & -59.880 & 119.71 & 124.7 & 5.08 & $U=6 \mathrm{eV}$ \\
& FM_HS_+2 & -65.697 & 117.70 & 145.9 & 4.34 & \\
& AFM_HS_+2 & -65.837 & 117.12 & 146.8 & 4.21 & \\
& FM_LS_+2 & -62.331 & 99.00 & 226.2 & 4.52 & \\
AFM_LS_+2 & -62.288 & 98.86 & 229.4 & 4.46 & \\
& FM_HS_+4 & -63.473 & 118.33 & 151.9 & 4.80 & $U=4 \mathrm{eV}$ \\
& FM_HS_+2 & -67.535 & 117.07 & 146.7 & 4.61 & \\
& AFM_HS_+2 & -67.788 & 115.98 & 150.1 & 4.17 & \\
& FM_LS_+2 & -67.524 & 97.88 & 245.5 & 4.60 & \\
AFM_LS_+2 & -67.471 & 97.87 & 247.3 & 4.46 & \\
\hline & & & & & & \\
& & & & \\
& & &
\end{tabular}


Table S3 The evolution of TM-O and O-O distances of pyrite-type $\mathrm{CrO}_{2}, \mathrm{MnO}_{2}$, $\mathrm{FeO}_{2}$ and $\mathrm{CoO}_{2}$ as a function of pressure.

\begin{tabular}{|c|c|c|c|c|c|}
\hline $\begin{array}{l}\text { Pressure } \\
(\mathrm{GPa})\end{array}$ & $D_{\mathrm{TM}-\mathrm{O}}(\AA)$ & $D_{\mathrm{O}-\mathrm{O}}(\AA)$ & $\begin{array}{l}\text { Pressure } \\
(\mathrm{GPa})\end{array}$ & $D_{\mathrm{TM}-\mathrm{O}}(\AA)$ & $D_{\mathrm{O}-\mathrm{O}}(\AA)$ \\
\hline \multicolumn{3}{|c|}{$\mathrm{CrO}_{2}$} & \multicolumn{3}{|c|}{$\mathrm{MnO}_{2}$} \\
\hline 0 & 1.966 & 2.403 & 0 & 1.967 & 2.671 \\
\hline 3.0 & 1.957 & 2.390 & 4.8 & 1.950 & 2.636 \\
\hline 10.7 & 1.938 & 2.362 & 11.4 & 1.931 & 2.595 \\
\hline 19.6 & 1.918 & 2.333 & 19.1 & 1.911 & 2.557 \\
\hline 29.9 & 1.898 & 2.304 & 28.0 & 1.892 & 2.512 \\
\hline 41.8 & 1.878 & 2.274 & 38.3 & 1.873 & 2.475 \\
\hline 55.4 & 1.859 & 2.239 & 50.3 & 1.854 & 2.433 \\
\hline 71.1 & 1.839 & 2.207 & 64.1 & 1.835 & 2.391 \\
\hline 89.1 & 1.820 & 2.174 & 80.2 & 1.816 & 2.351 \\
\hline 109.7 & 1.800 & 2.141 & 98.6 & 1.796 & 2.310 \\
\hline 133.3 & 1.781 & 2.107 & 119.8 & 1.777 & 2.269 \\
\hline 160.5 & 1.761 & 2.073 & 144.3 & 1.759 & 2.228 \\
\hline \multicolumn{3}{|c|}{$\mathrm{FeO}_{2}$} & \multicolumn{3}{|c|}{$\mathrm{CoO}_{2}$} \\
\hline 0 & 1.913 & 2.009 & 0 & 1.906 & 2.137 \\
\hline 8.2 & 1.893 & 1.991 & 2.3 & 1.901 & 2.132 \\
\hline 17.7 & 1.874 & 1.976 & 9.1 & 1.880 & 2.113 \\
\hline 28.6 & 1.854 & 1.958 & 16.9 & 1.867 & 2.094 \\
\hline 41.1 & 1.835 & 1.938 & 25.7 & 1.849 & 2.075 \\
\hline 55.5 & 1.816 & 1.914 & 35.7 & 1.832 & 2.056 \\
\hline 72.0 & 1.798 & 1.890 & 47.0 & 1.815 & 2.037 \\
\hline 90.8 & 1.779 & 1.864 & 59.8 & 1.798 & 2.020 \\
\hline 112.4 & 1.761 & 1.836 & 74.2 & 1.781 & 2.001 \\
\hline 124.3 & 1.751 & 1.822 & 90.5 & 1.764 & 1.983 \\
\hline 137.1 & 1.742 & 1.807 & 108.9 & 1.746 & 1.964 \\
\hline \multirow[t]{2}{*}{150.7} & 1.733 & 1.792 & 129.6 & 1.729 & 1.944 \\
\hline & & & 152.9 & 1.712 & 1.925 \\
\hline
\end{tabular}


Table S4 Results of SSAdNDP calculations. The calculations are performed at $100 \mathrm{GPa}$ for the pure pyrite-type (py) $\mathrm{AO}_{2}$ compounds, and at $0 \mathrm{GPa}$ for $\mathrm{AO}$ and the rutile-type (r) $\mathrm{AO}_{2}$ compounds. For $\mathrm{Mn}$-doped or Co-doped $\mathrm{FeO}_{2}$, the simulation is conducted at a fixed volume of $18.23 \AA^{3} /$ f.u. corresponding to that of $\mathrm{FeO}_{2}$ at $150 \mathrm{GPa}$. LP and $\mathrm{ON}$ represent the lone pair and occupation number, respectively. Previous calculated results are also included for comparison. ${ }^{1}$

\begin{tabular}{|c|c|c|c|c|}
\hline Phase & $\begin{array}{l}\text { Number of } 3 d \\
\text { unpaired electrons } \\
\text { on each cation }\end{array}$ & $\begin{array}{l}\text { Number of } \\
3 d \text { LPs on } \\
\text { each cation }\end{array}$ & $\begin{array}{l}\text { Number of } \\
\text { LPs on each O } \\
\text { and ON }(|\mathrm{e}|)\end{array}$ & $\begin{array}{l}\text { Number of } 2 \mathrm{c}- \\
2 \mathrm{e} \text { O-O bonds } \\
\text { per unit-cell }\end{array}$ \\
\hline \multicolumn{5}{|c|}{ pyrite-type $(\mathrm{Pa} \overline{\mathbf{3}}) \mathrm{TMO}_{2}$} \\
\hline $\mathrm{CrO}_{2}$ & 2 & 0 & $4,1.50-1.56$ & 0 \\
\hline $\mathrm{MnO}_{2}$ & 3 & 0 & $4,1.44-1.80$ & 0 \\
\hline $\mathrm{FeO}_{2}$ & 0 & 3 & $3,1.63-1.71$ & 4 \\
\hline $\mathrm{CoO}_{2}$ & 1 & 3 & $3,1.65-1.71$ & 4 \\
\hline \multirow[t]{2}{*}{$\mathrm{Mn}-\mathrm{FeO}_{2}$} & $3(\mathrm{Mn})$ & $0(\mathrm{Mn})$ & $4,1.48-1.66$ & 0 \\
\hline & $2(\mathrm{Fe})$ & $1(\mathrm{Fe})$ & & \\
\hline \multirow[t]{2}{*}{$\mathrm{Co}-\mathrm{FeO}_{2}$} & $1(\mathrm{Co})$ & $3(\mathrm{Co})$ & $3,1.56-1.68$ & 4 \\
\hline & $0(\mathrm{Fe})$ & $3(\mathrm{Fe})$ & & \\
\hline \multicolumn{5}{|c|}{ rutile-type $\left(P 4_{2} / \mathrm{mnm}\right) \mathrm{TMO}_{2}$} \\
\hline $\mathrm{CrO}_{2}$ & 2 & 0 & $4,1.50-1.68$ & 0 \\
\hline $\mathrm{MnO}_{2}$ & 3 & 0 & $4,1.52-1.81$ & 0 \\
\hline $\mathrm{FeO}_{2}$ & 4 & 0 & $4,1.64-1.80$ & 0 \\
\hline \multicolumn{5}{|c|}{$F m \overline{\mathbf{3}} m$ TMO } \\
\hline $\mathrm{MnO}$ & 5 & 0 & $4,1.80-1.84$ & 0 \\
\hline $\mathrm{FeO}$ & 4 & 1 & $4,1.78-1.85$ & 0 \\
\hline $\mathrm{CoO}$ & 3 & 2 & $4,1.72-1.86$ & 0 \\
\hline \multicolumn{5}{|c|}{ Others } \\
\hline py- $\mathrm{MgO}_{2}$ & 0 & 0 & $3,1.78-1.87$ & 4 \\
\hline$F m \overline{\mathbf{3}} m-\mathrm{MgO}$ & 0 & 0 & $4,1.68-1.83$ & 0 \\
\hline $\begin{array}{l}F m \overline{\mathbf{3}} m-\mathrm{MgO} \\
\text { [ref 1] }\end{array}$ & 0 & 0 & $4,1.76-1.86$ & 0 \\
\hline $\mathrm{r}-\mathrm{SiO}_{2}$ & 0 & 0 & $4,1.66-1.93$ & 0 \\
\hline py- $\mathrm{SiO}_{2}$ & 0 & 0 & $4,1.67-1.94$ & 0 \\
\hline
\end{tabular}


Table S5 The valence state of cations and $O$ anion based on the Bader charge analysis.

The calculations are performed at $100 \mathrm{GPa}$ for the pure pyrite-type (py) $\mathrm{AO}_{2}$ compounds, and at $0 \mathrm{GPa}$ for $\mathrm{AO}$ and the rutile-type (r) $\mathrm{AO}_{2}$ compounds. For Mn-doped or Co-doped $\mathrm{FeO}_{2}$, the simulation is conducted at a fixed volume of $18.23 \AA^{3} /$ f.u. corresponding to that of $\mathrm{FeO}_{2}$ at $150 \mathrm{GPa}$. The valence state of the previously predicted $I 4 / m m m \mathrm{FeO}_{2}$ or $\mathrm{CrO}_{2}$ is calculated at $1720 \mathrm{GPa}$ and $463 \mathrm{GPa}$, respectively. Previous calculated results are also included for comparison. ${ }^{2-4}$

\begin{tabular}{llll}
\hline Phase & $\begin{array}{l}\text { Charge } \\
\text { cation }\end{array}$ & $\begin{array}{c}\text { of } \\
\text { of anion }\end{array}$ & $\begin{array}{l}\text { Method \& } \\
\text { References }\end{array}$ \\
\hline \multicolumn{4}{c}{ pyrite-type $(\boldsymbol{P a} \overline{\mathbf{3}})$} \\
$\mathrm{TMO}$
\end{tabular}




\begin{tabular}{lccl}
\hline & \multicolumn{3}{c}{ Others } \\
\hline py- $\mathrm{MgO}_{2}$ & 1.68 & -0.84 & PBE \\
$I 4 / m c m-\mathrm{MgO}_{2}$ & 1.74 & -0.87 & PBE [ref 4] \\
$\boldsymbol{F} \boldsymbol{m} \overline{\mathbf{3}} \boldsymbol{m}-\mathrm{MgO}$ & 1.70 & -1.70 & PBE \\
& 1.66 & -1.66 & PBE [ref 3] \\
$\mathrm{qtz}_{-} \mathrm{SiO}_{2}$ & 3.22 & -1.61 & $\mathrm{PBE}$ \\
$\mathrm{r}-\mathrm{SiO}_{2}$ & 3.22 & -1.61 & $\mathrm{PBE}$ \\
$\mathrm{py}-\mathrm{SiO}_{2}$ & 3.26 & -1.63 & $\mathrm{PBE}$ \\
$\mathrm{I} / \mathrm{mmm}-\mathrm{FeO}_{2}$ & 1.20 & -0.60 & $U=5 \mathrm{eV}, 1720 \mathrm{GPa}$ \\
$I 4 / m m m-\mathrm{CrO}_{2}$ & 1.62 & -0.81 & $U=3 \mathrm{eV}, 463 \mathrm{GPa}$ \\
\hline
\end{tabular}


Table S6 Calculated parameters of the third-order Birch-Murnaghan equation of state (energy per formula unit $E_{0}$, volume per formula unit $V_{0}$, bulk modulus $K_{0}$ and its pressure derivative $\mathrm{K}_{0}$ ' at zero pressure) of $\mathrm{CoO}_{2}$ or $\mathrm{CoO}_{2} \mathrm{H}$.

\begin{tabular}{lllll}
\hline Phase & $\begin{array}{l}E_{0} / \text { f.u. } \\
(\mathrm{eV})\end{array}$ & $\begin{array}{l}V_{0} / \text { f.u. } \\
\left(\AA^{3}\right)\end{array}$ & $\begin{array}{l}K_{0} \\
(\mathrm{GPa})\end{array}$ & $K_{0}{ }^{3}$ \\
\hline $\mathrm{CaCl}_{2}$-type $\mathrm{CoO}_{2}$ & -15.765 & 25.84 & 205.5 & 4.78 \\
$2 \mathrm{H}-\mathrm{CoO}_{2} \mathrm{H}$ & -21.418 & 31.32 & 198.0 & 3.83 \\
$3 \mathrm{R}-\mathrm{CoO}_{2} \mathrm{H}$ & -21.425 & 31.31 & 199.5 & 3.76 \\
\hline
\end{tabular}


Table S7 Calculated lattice parameters and atomic coordinates of $\mathrm{CoO}_{2}$ and $\mathrm{CoO}_{2} \mathrm{H}$ under different conditions. The previous experimental ${ }^{5,6}$ and theoretical ${ }^{7,8}$ data are given for comparison.

\begin{tabular}{|c|c|c|c|c|c|c|c|c|c|c|}
\hline \multirow{2}{*}{ Phase } & \multirow{2}{*}{$\begin{array}{l}\text { Pressure } \\
(\mathrm{GPa})\end{array}$} & \multirow{2}{*}{$\mathrm{a}(\AA)$} & \multirow{2}{*}{$\mathrm{b}(\AA)$} & \multirow{2}{*}{$c(\AA)$} & \multirow{2}{*}{ Site } & \multirow{2}{*}{$\begin{array}{l}\text { Wyckoff } \\
\text { symbol }\end{array}$} & \multicolumn{3}{|c|}{ internal coordinates } & \multirow{2}{*}{$\begin{array}{l}\text { Method and } \\
\text { reference }\end{array}$} \\
\hline & & & & & & & $\mathrm{X}$ & $\mathrm{y}$ & $\mathrm{Z}$ & \\
\hline $\mathrm{CoO}_{2-}$ & \multirow{2}{*}{3.3} & \multirow{2}{*}{4.569} & \multirow{2}{*}{3.996} & \multirow{2}{*}{2.874} & $\mathrm{Cr}$ & $2 \mathrm{a}$ & 0 & 0 & 0 & \multirow[t]{2}{*}{ this study } \\
\hline Pnnm & & & & & $\mathrm{O}$ & $4 g$ & 0.3586 & 0.2360 & 0 & \\
\hline $\mathrm{CoO}_{2-}$ & \multirow{2}{*}{0} & \multirow{2}{*}{4.41} & \multirow{2}{*}{4.10} & \multirow{2}{*}{2.85} & $\mathrm{Cr}$ & $2 \mathrm{a}$ & 0 & 0 & 0 & \multirow{2}{*}{$\begin{array}{l}U=4.91 \mathrm{eV} \\
{[\text { ref 7] }}\end{array}$} \\
\hline Pnnm & & & & & $\mathrm{O}$ & $4 g$ & 0.34 & 0.27 & 0 & \\
\hline $\mathrm{CoO}_{2} \mathrm{H}-$ & \multirow{3}{*}{1.6} & \multirow{3}{*}{2.873} & \multirow{3}{*}{2.873} & \multirow{3}{*}{8.694} & $\mathrm{Co}$ & $2 \mathrm{a}$ & 0 & 0 & 0 & \multirow[t]{3}{*}{ this study } \\
\hline $\mathrm{P6}_{3} / m m c$ & & & & & $\mathrm{O}$ & $4 f$ & $1 / 3$ & $2 / 3$ & 0.1113 & \\
\hline$(2 \mathrm{H})$ & & & & & $\mathrm{H}$ & $2 \mathrm{c}$ & $1 / 3$ & $2 / 3$ & 0.25 & \\
\hline $2 \mathrm{H}$ & 0 & 2.855 & 2.855 & 8.805 & --- & --- & $\begin{array}{ll}-- \\
\end{array}$ & $\begin{array}{ll}-- \\
\end{array}$ & $\begin{array}{ll}-- \\
\end{array}$ & Exp. [ref 5] \\
\hline $2 \mathrm{H}$ & 0 & 2.844 & 2.844 & 8.551 & --- & --- & --- & --- & --- & LDA [ref 8] \\
\hline $2 \mathrm{H}$ & 0 & 2.933 & 2.933 & 8.723 & --- & --- & --- & --- & --- & PBE [ref 8] \\
\hline $2 \mathrm{H}$ & 0 & 3.030 & 3.030 & 8.878 & --- & --- & --- & --- & --- & $\begin{array}{l}\mathrm{LDA}+U \\
{[\text { ref 8] }}\end{array}$ \\
\hline $\mathrm{CoO}_{2} \mathrm{H}-$ & & & & & $\mathrm{Co}$ & $3 a$ & 0 & 0 & 0 & this study \\
\hline$R-3 \mathrm{~m}$ & 3.0 & 2.865 & 2.865 & 13.020 & $\mathrm{O}$ & $6 c$ & 0 & 0 & 0.4077 & \\
\hline$(3 \mathrm{R})$ & & & & & $\mathrm{H}$ & $3 b$ & 0 & 0 & 0.5 & \\
\hline $3 \mathrm{R}$ & 0 & 2.855 & 2.855 & 13.157 & --- & --- & --- & --- & $\begin{array}{ll}-- \\
-\end{array}$ & Exp. [ref 6] \\
\hline $3 R$ & 0 & 2.844 & 2.844 & 12.825 & --- & -- & --- & --- & --- & LDA [ref 8] \\
\hline $3 R$ & 0 & 2.933 & 2.933 & 13.080 & --- & --- & --- & --- & --- & PBE [ref 8] \\
\hline $3 R$ & 0 & 2.843 & 2.843 & 12.854 & --- & --- & --- & --- & --- & $\begin{array}{l}\mathrm{LDA}+U \\
{[\text { ref 8] }}\end{array}$ \\
\hline
\end{tabular}




\section{Supplementary references}

(1) Popov, I. A.; Jimenez-Izal, E.; Alexandrova, A. N.; Boldyrev, A. I. Multicenter Bonding Effects in Oxygen Vacancy in the Bulk and on the Surface of MgO. J Phys. Chem. C 2018, $122,11933-11937$.

(2) Liu, J.; Hu, Q.; Bi, W.; Yang, L.; Xiao, Y.; Chow, P.; Meng, Y.; Prakapenka, V. B.; Mao, H. K.; Mao, W. L. Altered Chemistry of Oxygen and Iron under Deep Earth Conditions. Nat. Commun. 2019, 10, 153.

(3) Lu, C.; Amsler, M.; Chen, C. Unraveling the Structure and Bonding Evolution of the Newly Discovered Iron Oxide $\mathrm{FeO}_{2}$. Phys. Rev. B 2018, 98, 054102.

(4) Zhu, Q.; Oganov, A. R.; Lyakhov, A. O. Novel Stable Compounds in the Mg-O System under High Pressure. Phys. Chem. Chem. Phys. 2013, 15, 7696-7700.

(5) Deliens, M.; Goethals, H. Polytypism of Heterogenite. Mineral. Mag. 1973, 39, 152157.

(6) Burlet, C.; Goethals, H.; Vanbrabant, Y. Delafossite Structure of Heterogenite Polytypes $\left(\mathrm{HCoO}_{2}\right)$ by Raman and Infrared Micro-Spectroscopy. Spectrochim Acta Part A: Mol. Biomol. Spectrosc. 2016, 159, 90-97.

(7) Wang, S.; Liu, J.; Qie, Y.; Gong, S.; Sun, Q.; Jena, P. Discovery of a High-Pressure Phase of Rutile-Like $\mathrm{CoO}_{2}$ and its Potential as a Cathode Material. J. Mater. Chem. A 2018, 6, 18449-18457.

(8) Upadhyay, D.; Pratap, A.; Jha, P. K. A First Principles Study on Structural, Dynamical, and Mechanical Stability of Newly Predicted Delafossite $\mathrm{HCoO}_{2}$ at High Pressure. $J$. 
Raman Spectrosc. 2019, 50, 603-613. 